\title{
The development of a community-based medical education program in Korea
}

\author{
Jung Eun Yoo', Seo Eun Hwang ${ }^{2}$, Gyeongsil Lee ${ }^{3}$, Seung Jae Kim ${ }^{2,4}$, Sang Min Park ${ }^{2,5}$, Jong-Koo Lee, ${ }^{2,5}$ \\ Seung-Hee Lee, Hyun Bae Yoon ${ }^{6}$ and Ji Eun Lee \\ 'Department of Family Medicine, Samsung Medical Center, Sungkyunkwan University School of Medicine, \\ ${ }^{2}$ Department of Family Medicine, Seoul National University Hospital, ${ }^{3}$ Department of Family Medicine, Health \\ Promotion Center, Chung-Ang University Hospital, ${ }^{4}$ Department of Medical Education, Seoul National University \\ College of Medicine, ${ }^{5}$ Department of Family Medicine, Seoul National University College of Medicine, Seoul, \\ ${ }^{6}$ Office of Medical Education, Seoul National University College of Medicine, and ${ }^{7}$ Department of Family Medicine, \\ CHA Bundang Medical Center, CHA University, Seongnam, Korea
}

Purpose: The introduction of community-based medical education would help improve the quality of primary care. This paper suggests learning objectives and an educational program for community-based medical education.

Methods: The educational program was developed in a 1-day consensus workshop. Twenty experts, including faculty members from family medicine department of a college of medicine in Seoul and community-based preceptors, participated in the program. A needs-assessment survey was conducted among community-based preceptors before the workshop. Through this workshop, we derived learning objectives and a standardized curriculum for community-based medical education.

Results: In the questionnaire before the workshop, community-based preceptors voiced concerns over the program's potential costs and the time required for teaching. The learning objectives and educational programs derived from the workshop's consensus were consistent with the characteristics of the primary care. Based on the results of this workshop, the joint expert team developed a standard educational program on two core topics: clinical teaching and mentoring.

Conclusion: From this curriculum development process, participants could construct a more standardized curriculum for community-based medical education. Future studies are needed to evaluate the long-term outcomes of these educational programs, such as the learners' satisfaction and achievement.

Key Words: Primary health care, Preceptorship, Consensus, Educational models, Curriculum

\section{Introduction}

As healthcare becomes more specialized and fragmented, patients seeking treatment in the current healthcare system are facing more problems. Medical services are not well-coordinated, and patients often consult multiple doctors about common health problems. On the other hand, it is sometimes difficult for patients to know which doctor to visit for help. Medical expenditures continue to rise and are becoming a financial strain, and there are regions where access to medical care is lacking.

Although primary healthcare targets the health
Received: June 12, 2018 • Revised: July 23, 2018 • Accepted: September 11, 2018 Corresponding Author: Ji Eun Lee (https://orcid.org/0000-0003-3171-1713) Department of Family Medicine, CHA Bundang Medical Center, CHA University, 59 Yatap-ro, Bundang-gu, Seongnam 13496, Korea

Tel: +82.31.780.5000 email: jieun10@gmail.com
Korean J Med Educ 2018 Dec; 30(4): 309-315.

https://doi.org/10.3946/kjme.2018.105

eISSN: 2005-7288

(C) The Korean Society of Medical Education. All rights reserved. This is an open-access article distributed under the terms of the Creative Commons Attribution Non-Commercial License (http:// creativecommons.org/licenses/by-nc/3.0/), which permits unrestricted non-commercial use, distribution, and reproduction in any medium, provided the original work is properly cited. 
problems of most of the population, experts on medical education, research, health policy, and medical finance have not paid much attention to the effectiveness of primary care in preventing illness and premature death. However, the importance of primary care in meeting the needs of individuals, families, communities, and nations has become more apparent in recent years. According to previous research, 750 people per 1,000 adults have experienced at least one symptom during a month [1]. Of these 750 adults, 250 were consulted by doctors, five were referred to other specialists, and only one was admitted to a university hospital [1]. Forty years later, the same research procedure was repeated, showing that the use of healthcare was similar despite significant changes in medical institutions and spending [2]. The number of primary care users was 12 times higher than that of tertiary hospital users, and a significant portion of healthcare was provided through primary care, self-care, and outpatient care [2]. These results indicate that most health problems experienced by the population could not be detected and properly managed without primary care.

In Korea, as the importance of primary care physicians has been underestimated, the percentage of physicians who have received systematic primary medical training has remained low [3]. As a result, a decline in the quality of medical services provided by primary care has been observed [4].

In order to improve the quality of primary care services, a curriculum that cultivates primary medical care capacity through medical school education is needed. Until now, most medical education programs have consisted of simple memorization and observationoriented clinical practice [5], though there has been an effort to introduce a more practical program for the medical curriculum. For example, the Korea Medical Licensing Examination introduced the clinical perfor- mance examination and objective structured clinical examination in 2010. Through the process of preparing for practical examinations, the medical students can have good opportunities for clinical practice during the regular curriculum, as well as improving their skills of communication with patients and their physical examination ability [6].

However, the participation of clinical practice in the tertiary hospital is limited because the disease distribution of the target patient group differs from the primary medical reality. There are also a growing number of restrictions on the use of patients' personal information. For these reasons, there is constant discussion of the need for the reorganization of the medical curriculum.

Since the 1970s, the ward-based medical education of tertiary hospitals has gradually been transformed into a community-based outpatient practice. This is called community-based medical education (CBME). The effectiveness of the CBME curriculum has been extensively researched. If CBME is introduced in Korea and the primary care practice in the community is revitalized, the quality of primary care could be improved by educating primary care physicians on firstcontact care, continuity, comprehensive care, coordinative function and personalized care.

The purpose of this study is to suggest a basic model for CBME in Korea, based on the opinions of community preceptors and medical education experts. We also identify the anticipated difficulties in applying CBME model in Korea.

\section{Methods}

We held a "Capacity Building Workshop for Community Preceptors," centering on medical education 
specialists and community medical education preceptors. The workshop consisted of lectures on strengthening preceptor capacity, the consensus process for learning objectives, and designing curriculums for CBME. In addition, we conducted a preliminary questionnaire before the workshop, in order to understand the difficulties and needs of community medical education preceptors. The present study was approved by the institutional review board of the Seoul National University Hospital Biomedical Research Institute (IRB approval no., C-1803-117-932). The Board waived the requirement of informed consent.

\section{Preliminary questionnaire}

To assess preceptor's needs or opinions about CBME, we conducted a preliminary survey. We used the preliminary questionnaire which was developed for this workshop by two medical education experts. At first, medical education experts reviewed similar articles and summarized questionnaires to meet our situations. Second, we confirmed the preliminary questionnaires through discussion. Finally, it included the following items: (1) the required abilities for students to participate in CBME; (2) the concerns of practitioners involved in community-based teaching; and (3) the prerequisites for $\mathrm{CMBE}$ preceptors. The preliminary questionnaire was administered to 11 preceptors for community medical education.

\section{The consensus workshop}

\section{1) The definition of 'consensus workshop'}

The consensus workshop is composed of five simple steps: set a context, brainstorm in layers, cluster ideas, name the clusters, and resolve the names. All the process is conducted by facilitators. The facilitator draws on the ideas of the process through process design, process management, and conflict management, helping the workshop solve creative problems. The facilitator must be able to play an important role as the team builder, process checker, time keeper, and lecturer throughout the workshop [7].

\section{2) Details of the consensus workshop}

The workshop was held on February 12, 2017 at Seoul National University Medical Center International Hall. A total of 22 participants attended. Among them were 11 preceptors for community medical education, two professors in the medical education department at a college of medicine in Seoul, five professors in the family medicine department at a college of medicine in Seoul, and four fellows in the family medicine department at a college of medicine in Seoul.

The overall workshop was facilitated by a professor of the medical education department at a college of medicine in Seoul, who first proposed the concepts and methods and led discussion on specific subjects. The participants were divided into four subgroups and each group consisted of community preceptors and family medicine physicians from the university hospital. Facilitators instructed the brainstorming session so that it occurred individually first, the individual selected his or

Fig. 1. Process of Consensus Workshop

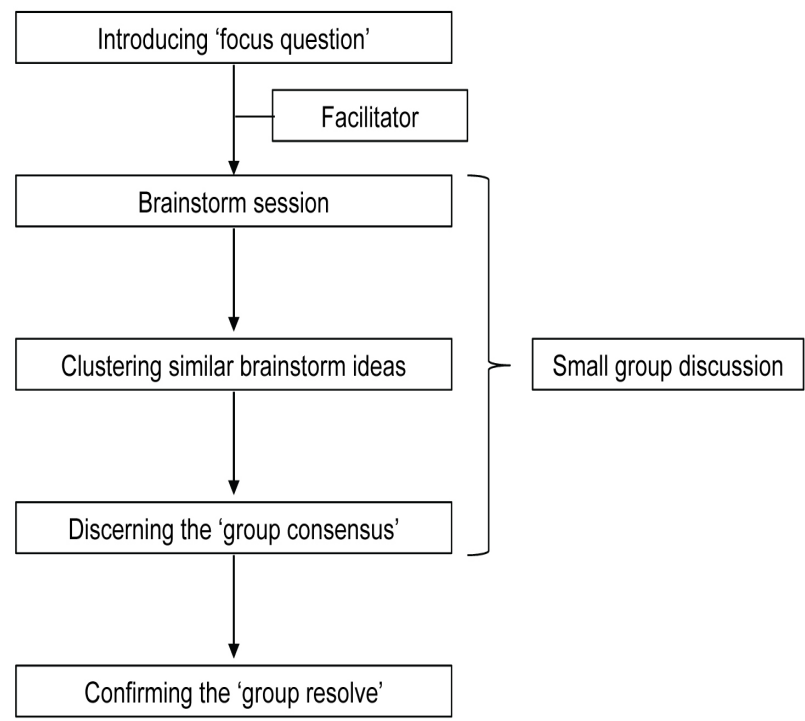


her favorites and then shared it with the group. After the subgroup discussion, we shared derived results regarding the learning goals and curriculum of CBME. We also discussed the each cluster of results in order to test our resolve and to check if this is what a whole group really think and what our next steps will be (Fig. 1).

\section{Results}

\section{Preliminary questionnaire analysis}

A total of 10 subjects (nine males and one female) participated in the preliminary questionnaire, and the response rate was $90.9 \%$. The average student teaching experience was 4.67 years, and the career distribution as a specialist was 6-10 years.

When asked about the abilities required for students to receive practical training in the community, $70 \%$ said that attitudes were important, and 30\% said that knowledge and clinical skills were important. The attitudes they referenced were enthusiasm for learning, courtesy, and a sincere attitude toward patients and staff. The knowledge and clinical skills the respondents referred to were related to understanding patients, students needed not be biased and are able to solve difficult medical problems in textbook cases.

The difficulties as a preceptor for CBME pointed to the need for prior cooperation of patients and hospitals. Other concerns included the potential cost and time required for teaching. There was also a lack of guidelines on standardized training materials, evaluation criteria, and training methods. In order to overcome these limitations, medical schools need to provide the materials necessary for student education and provide sufficient preliminary education for the students. Preceptors must have the competence required for edu- cation, enthusiasm, experience as a local doctor, a relationship with patients and local residents, and a variety of clinical experience and knowledge.

\section{Deriving learning objectives}

By sharing the results discussed in each subgroup, the following common learning objectives were derived.

\section{1) General learning objectives}

General learning objectives are (1) to understand the characteristics and approaches of patients in primary care; (2) to understand primary care systems; and (3) to understand the nature of family medicine and the role of family physicians.

\section{2) Specific learning objectives}

Specific learning objectives are as follows: (1) diagnostic approaches and treatment plans could be $\mathrm{e}^{-}$ stablished based on physical examinations, interviews, etc., for patients who are members of the community; (2) solve the health problems according to the life cycle by considering the patient's relationship with patients and family; (3) understand the structure of primary care and its management; and (4) think about desirable doctors and find role models.

\section{Deriving a standard curriculum}

We shared the results discussed in the four subgroups, and derived the following common goals of education.

1) Goals for students in required courses

Goals for students in required courses are (1) to provide preliminary notes using standard questions and perform preliminary examinations; (2) to perform the role of a medical assistant, such as blood pressure and blood glucose measurement; (3) to share the experience of dispatch training after the training session; (4) make a list of common diseases and how to treat them; (5) to participate in the practice after pre-acquisition of hypertension, diabetes, and hyperlipidemia, and conduct 
a basic knowledge evaluation before the practice if necessary; and (6) to conduct a preliminary consultation and observe patient education on smoking and drinking with the goal of lifestyle modification.

\section{2) Goals for students in elective courses}

Goals for students in elective courses are (1) to write a patient referral note; (2) to perform simple procedures such as dressing and reading X-ray; (3) to understand the process of a public inspection and implementation cycle; and (4) to have time to talk with the preceptors during lunch.

\section{Development of practical notes}

Based on the results of the workshop, we developed practical notes. A checklist of basic clinical skills and essential patient groups was developed to confirm basic clinical experience and to receive feedback from the preceptors. In addition, a case report was prepared for one patient, which included basic patient information such as address, current medical history, family history, and social history. This made it easier to listen to medical history and to train students on a series of treatment procedures from treatment planning to patient education. In addition, the prepared exercise notes were confirmed through preceptor feedback (Supplement 1).

\section{Discussion}

The purpose of this study is to introduce CBME and to present a basic model suitable for Korean medical education by developing learning goals and a curriculum. The need for community preceptors and their support programs, which play a key role in CBME, has also been examined.

There has been a worldwide increase of cost and inequalities in medical care. In Korea, there have been problems of high medical expenditure growth, medical imbalance, and inefficiency of medical expenditures [8]. To counteract this, we advocate for the reinforcement and improvement of primary care. It is particularly important to educate future medical students so that they are able to carry out the inclusiveness, sustainability, and coordination functions that are attributes of primary care. However, the current medical school curriculum is guided by the specialized physician, and this is insufficient in educating students about these components of primary care.

In North America, the need for CBME has emerged since the 1970s. CBME refers to medical education in which a student is directly exposed to the role of primary care through education about the community, and the patient's illness can be understood within the context of family, society, and community. In the 1970s, as the need for practical training in primary care increased, ward-based practice shifted to communitybased outpatient practice, and since the 1990s blockcycle practice has been expanding into community practice [9]. In addition, in the 2000s, prominent medical schools have introduced programs based on CBME and are gradually expanding [10]. Several studies have demonstrated the effectiveness of all this CBME. Compared to existing university-centered education, CBME has the effect of helping students understand diagnosis and communication skills, as well as their patients' illnesses within family and social contexts [11]. In Korea, there also has been a great effort to implement CBME curriculums. However, the main form of such programs currently being implemented is just dispatching students to local primary care facilities only once or twice a week. It is not enough to get the effect of CBME curriculums. A more intensive and systematic program would be required to achieve the goal of CBME. In this context, our study results could be an alternative model 
for medical education.

CBME requires a consistent educational philosophy and strategies to drive the quality of primary care education. To this end, Harvard Medical School mandates the involvement of preceptors in the areas of course management, teaching development and quality management [9]. Harvard Medical School also provides community preceptors with up-to-date information in new content areas, establishes common standards for teaching and evaluation, and runs programs to develop teaching skills [9]. The program is designed to provide a variety of workshops for all levels of students, held 8 times a year, and students take a separate workshop in each community education institution and medical school. One-on-one mentoring, newsletters and lecture notes are provided to help develop the educational capabilities of participating preceptors [9].

In this study, we also tried to solve the problems of CBME and strengthen the capacities of preceptors by communicating with educational experts in the workshop. A preliminary questionnaire suggested that community medical education preceptors need competence in education, enthusiasm for education, experience practicing medicine in the community, the ability to form relationships with patients and local residents, and clinical competence. On the other hand, the obstacles for preceptors were a lack of the following: educational materials and guidelines, standardized education methods, basic knowledge, and recognition as student educators. Another obstacle was that they were expected to provide all the education materials, educational objectives, and program composition themselves. Therefore, in order for community preceptors to strengthen their capacities and fully demonstrate their capabilities, it will be necessary to provide standardized educational materials and programs, as well as preceptor recognition and support, at the medical school level.
Through the consensus workshop in this study, a basic model of CBME was constructed by deriving the learning objectives and education items suitable for CBME. It is important to educate students about the concept, importance, and actual condition of primary care. It is also important to study scientific research or current knowledge, but emphasizing the patient-doctor relationship can be a factor to strengthen primary care and reinforce the importance of the preceptor as a role model. The learning objectives derived from this workshop were consistent with the inclusiveness, sustainability, and holistic approach that characterize primary care.

In order to achieve their learning goals, the participants of the workshop came to a consensus on the necessity of standardized practical notes. This research group developed practical notes reflecting the results of the workshop, and introduced them to their practice at the family medicine department of the college of medicine in Seoul. In the future, it will be necessary to evaluate the efficacy of applying this to practical medical school courses and to search for a development plan.

There were several limitations to our study. First, it was developed at a university college of medicine, and thus cannot be universally applied to all Korean medical schools. Furthermore, this study was carried out only with preceptors who were participating in a community medical education. Therefore, to prove the effectiveness of CBME, a comparative study for students applying our developed model is needed. Moreover, the quality of primary care medical education should be improved by expanding the length of CBME programs.

In conclusion, in this present study, we developed a CBME model suitable for Korean context with the help of medical education specialist and community clinical educators. By applying it to medical school education and seeking a development plan, it is expected to 
contribute to improve primary medical education.

\section{Supplementary Material}

Supplement 1. Community-based medical education: practical notes and checklists. Data files are available from https://doi.org/10.3946/kjme.2018.105.

\section{ORCID:}

Jung Eun Yoo: https://orcid.org/0000-0001-8344-6291; Seo Eun Hwang: https://orcid.org/0000-0001-6749-0970; Gyeongsil Lee: https://orcid.org/0000-0003-1910-9658; Seung Jae Kim: https://orcid.org/0000-0001-8885-7137; Sang Min Park: https://orcid.org/0000-0002-7498-4829; Jong-Koo Lee: https://orcid.org/0000-0003-4833-1178; Seung-Hee Lee: https://orcid.org/0000-0001-8672-5253; Hyun Bae Yoon: https://orcid.org/0000-0003-4367-5350; Ji Eun Lee: https://orcid.org/0000-0003-3171-1713

Acknowledgements: None.

Funding: None.

Conflicts of interest: No potential conflict of interest relevant to this article was reported.

Author contributions: all authors: (1) have made substantial contributions to conception and design, or acquisition of data, or analysis and interpretation of data; (2) have been involved in drafting the manuscript or revising it critically for important intellectual content; and (3) have given final approval of the version to be published.

\section{References}

1. White KL, Williams TF, Greenberg BG. The ecology of medical care. N Engl J Med. 1961;265:885-892.

2. Green LA, Fryer GE Jr, Yawn BP, Lanier D, Dovey SM. The ecology of medical care revisited. N Engl J Med. 2001;344(26):2021-2025.

3. Kim Y. Status and prospect of medical clinics in health system. Healthc Policy Forum. 2012;10:16-23.

4. Lee JH. Primary care and family medicine. In: Korean Academy of Family Medicine, ed. Textbook of Family Medicine. Seoul, Korea: Hankook Medicine; 2008.

5. Kim K, Lee S, Park H. Reforming medical education for strengthening primary care. J Korean Med Assoc. 2013; 56(10):891-898.

6. Park HK. The impact of introducing the Korean Medical Licensing Examination clinical skills assessment on medical education. J Korean Med Assoc. 2012;55(2): 116-123.

7. Hori K. Facilitation skill training. Hyun $\mathrm{CH}$, trans-ed. Seoul, Korea: Ilbit; 2005.

8. Ministry of Health and Welfare. Korean national health accounts and total health expenditure in 2010. Sejong, Korea: Ministry of Health and Welfare; 2011.

9. Ogur B, Hirsh D. Learning through longitudinal patient care: narratives from the Harvard Medical SchoolCambridge Integrated Clerkship. Acad Med. 2009;84(7): 844-850.

10. Kelly L, Walters L, Rosenthal D. Community-based medical education: is success a result of meaningful personal learning experiences? Educ Health (Abingdon). 2014;27(1):47-50.

11. Walters L, Greenhill J, Richards J, et al. Outcomes of longitudinal integrated clinical placements for students, clinicians and society. Med Educ. 2012;46(11):10281041 . 